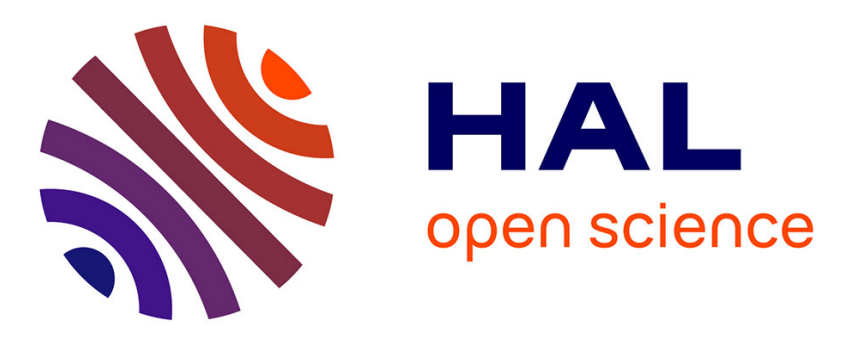

\title{
Structural studies of the q1 charge-density-wave in $\mathrm{NbSe} 3$
}

\author{
A. Moudden, L. Vasiliu-Doloc, P. Monceau, F. Levy
}

\section{To cite this version:}

A. Moudden, L. Vasiliu-Doloc, P. Monceau, F. Levy. Structural studies of the q1 chargedensity-wave in NbSe3. Journal de Physique IV Proceedings, 1993, 03 (C2), pp.C2-121-C2-124. 10.1051/jp4:1993223 . jpa-00251306

\section{HAL Id: jpa-00251306 https://hal.science/jpa-00251306}

Submitted on 1 Jan 1993

HAL is a multi-disciplinary open access archive for the deposit and dissemination of scientific research documents, whether they are published or not. The documents may come from teaching and research institutions in France or abroad, or from public or private research centers.
L'archive ouverte pluridisciplinaire HAL, est destinée au dépôt et à la diffusion de documents scientifiques de niveau recherche, publiés ou non, émanant des établissements d'enseignement et de recherche français ou étrangers, des laboratoires publics ou privés. 


\title{
Structural studies of the $q_{1}$ charge-density-wave in $\mathrm{NbSe}_{3}$
}

\author{
A.H. MOUDDEN, L. VASILIU-DOLOC, P. MONCEAU* and F. LEVY* ${ }^{* *}$ \\ Laboratoire Léon Brillouin, CEA-CNRS, CE/Saclay, 91191 Gif-sur-Yvette, France \\ ${ }^{*}$ C.R.T.B.T., CNRS, BP. 166X, 38042 Grenoble cedex, France \\ ** Institut de Physique Appliquée, E.P.F., 1015 Lausanne, Switzerland
}

$\mathrm{X}$-ray scattering studies of $\mathrm{NbSe}_{3}$ show : i) that the $q_{1}$ charge density wave (CDW) fluctuations correspond to quasi-two-dimensional correlations with an inplane anisotropy $\xi_{b^{*}} / \xi_{a^{*}} \sim 3.5$. ii) The wave vector $q_{1}$ has a significant temperature variation accounted for by an activation energy of about $600 \mathrm{~K}$. iii) Higher order $(n=2,3)$ harmonics are evidenced. Their temperature variation shows a clear saturation of the intensities at onset temperatures decreasingly smaller when increasing $\mathrm{n}$. The results suggest the presence of an Ising like feature underlying the CDW .

After nearly 20 years of continued interest ${ }^{1}$ on $\mathrm{NbSe}_{3}$, the question whether this system should be described in the weak or strong electron-phonon coupling limit is still debated. There is however, an increasing number of detailed experiments ${ }^{2,3}$ that quantitatively disagree with the predictions of the mean field Peierls-Fröhlich model in the weak coupling limit. Whether these discrepancies are the effects of fluctuations ${ }^{4}$ or the effects of strong electron-phonon coupling, is yet to be understood. In this paper we report on three structural aspects of the CDW $q_{1}$. We will show that there is a modest anisotropy of the CDW correlations and a strong temperature dependence of the wave vector (strong when compared to the prediction of the mean field small coupling limit). We further show the existence of harmonics with intensity saturation which seems to be in favour of the strong coupling limit. The X-ray scattering experiments were carried out on the $\mathrm{X} 22 \mathrm{C}$ beam line at the National Synchrotron Light Source of Brookhaven. $1.7 \AA$ X-rays from a Ge(111) double monochromator were used with a $\mathrm{Ge}(111)$ analyzer to achieve a very good momentum resolution. We have measured the thermal variation of the scattering profile along the three directions of the reciprocal lattice as described earlier ${ }^{3}$. The result for the inverse correlation lengths corrected from the instrumental 
resolution function, is shown on the left side of the next figure.
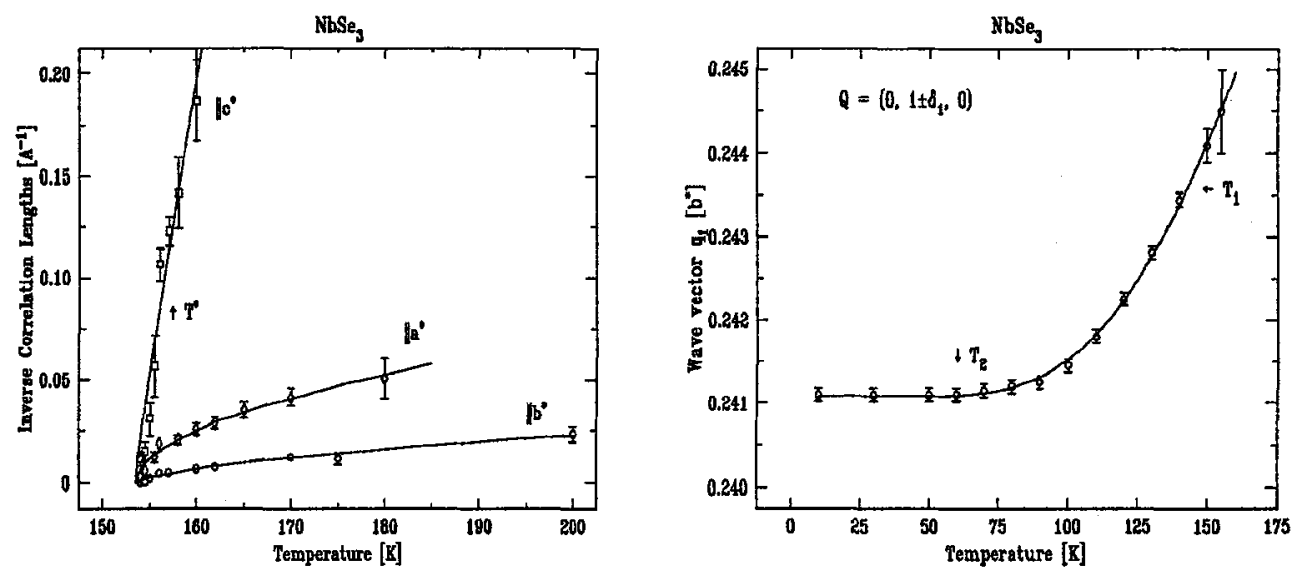

The solid lines are parametrization with a power law behaviour of the form $\xi^{-1} \sim\left(T-T_{1}\right)^{\nu}$. We find for the in-plane anisotropy $\xi_{b^{*}} / \xi_{a^{*}}=3.5 \pm 0.5$ and for the out-of-plane $\xi_{b^{*}} / \xi_{\mathrm{c}^{*}}=27.0 \pm 2$. From the measured anisotropies it is clear that $\mathrm{NbSe}_{3}$ is a quasi-2D, and it is more appropriate to analyse the observed CDW fluctuations in terms of weakly coupled layers. In this case the mean field treatment leads to the $3 D$ coupling constant $\lambda=\chi_{3 D}^{-1}\left(T^{*}\right)$, where $T^{*}$ is the $2 \mathrm{D} / 3 \mathrm{D}$ crossover temperature. With a proper normalization of the diffuse scattering intensity $I_{D}(q) \sim S(q)=k T \cdot \chi_{3}(q)$ and using the previous results that the longitudinal component of the distortion wave $q_{1}$ is dominated by the displacement of $\mathrm{Nb}$ atoms on the yellow chains with an amplitude of about $2 \%$, we find the coupling energy $\lambda=30 \pm 5 K$.

As the temperature is decreased below $\sim 150 \mathrm{~K}$, the scattering peak becomes narrower and increases in intensity. The critical wave vector given by the satellite position is shifted far away from the commensurate value of $1 / 4$ as shown in the previous right side figure. Below $\sim 80 \mathrm{~K} q_{1}(T)$ remains constant at about $0.2411(1)$ for which the best rational approximant is $7 / 29$. In the picture of bonding 5 involving every second pair of $\mathrm{Nb}$ atoms along the chains with the shortest Se-Se pairing, this basic period would correspond to one missing bond every seven periods. This distance increases up to 11 periods close to the transition temperature. In this scheme one expects a step-like variation for $q_{1}(T)$, since it should reflect the number of missing bonds per chain. Our measured variation of $q_{1}(T)$ does not reveal such steps which might be too small to be detected with the present resolution. However $q_{1}(T)$ is 
interestingly well accounted for by an activated variation $q_{1}(T)=q_{1}(0)+C \times e^{-(\Delta E / T)}$, with $\Delta E \approx 600 K$. Similar results have been reported for blue bronzes ${ }^{6}$ and attributed first to a specific band structure of the blue bronzes. Our present results suggest that the activation form is very likely to be a common feature of the CDW systems. Futher, we have measured in some details the integrated intensities of the first, second and third order satellite peaks. Their temperature evolution is reported on the next figure.

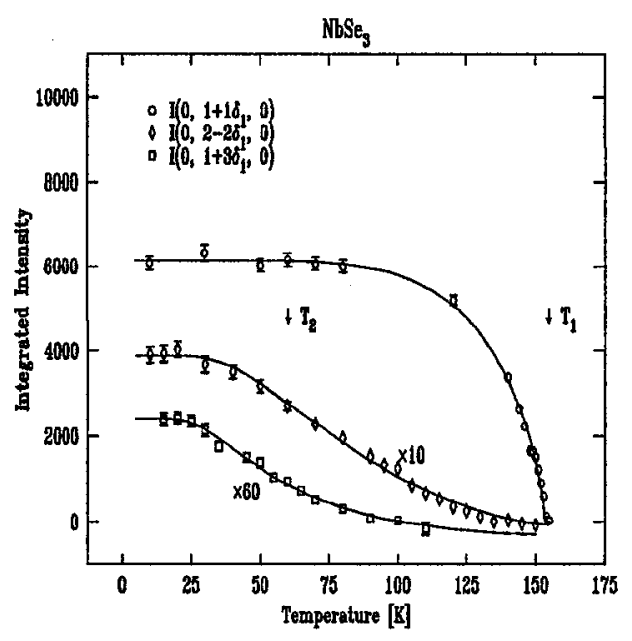

Clearly the intensity of the first harmonic saturates below $\sim 110 \mathrm{~K}$ while the second and third satellites saturate at lower temperatures 40 and $25 \mathrm{~K}$ respectively. Notice that when crossing the $q_{2}$-CDW transition at about $60 \mathrm{~K}$ there is no anomaly in none of these quantities nor in the wave vector variation consistent with two independent CDW systems. The observation of higher harmonics may be ascribed to higher order diffraction from a single sine wave distortion.

In this case one expects to see the intensity of the $n^{\text {th }}$ order satellite to follow (with $\mathrm{n}$ times the exponent) the temperature dependence of the fundamental peak $\mathrm{n}=1$. Obviously, this is not the case. If we examine the temperature range from $110 \mathrm{~K}$ to $40 \mathrm{~K}$ we can qualitatively see that while the intensity of the first order satellite is saturated the second and third order are still strongly increasing. Quantitatively we have checked that when we use the atomic displacement obtained from the analysis of the set of first order satellite only, and compute the intensities of the second order satellites we find that the contamination is less than $30 \%$.

One of the important ingredient of the Peierls instability is the divergence of the electronic susceptibility because of the nesting property of the Fermi surface. In a quasi-2D system such as $\mathrm{NbSe}_{3}$ with modest anisotropy and then a closed Fermi surface, the electronic susceptibility will apriori show not even a definite maximum. Thus, in order to occur, the instability will require a stronger electronphonon coupling. In this case the critical wave vector will be determined partly by 
the electronic susceptibility but also by the $q$ dependence of the coupling constant and eventually by the phonon dispersion. Mean field calculations ${ }^{7}$ of the Peierls chain have been done using a quadratic expansion of the electronic dispersion to avoid the electron-hole symmetry introduced by the standard linearization. With an appropriate cut off and a finite slope of the phonon branch, the authors show that most of the experimentally observed thermal variation of the wave vector, can be accounted for by this model. However more recent studies ${ }^{8}$ of the same model but using the explicit tight binding electronic dispersion with no cut-off, demonstrate complete disagreement in magnitude and sometimes in sign with the experimental observations and the authors propose an alternating strong electron-phonon coupling. It has been shown ${ }^{9}$ that above some critical value of the coupling constant a transition by breaking of analiticity occurs, and leads to the formation of electron pairs pinned to the lattice (bipolarons). The incommensurate CDW must be viewed as an array of bipolarons and the Peierls transition as a gradual melting of the bipolaron lattice. The ground state and the excitations of this bipolaronic CDW are shown to be described by an interacting pseudo-spin Hamiltonian. The presence of the harmonics indicate a genuine deviation of the CDW from a sine wave. The observed saturations at temperatures depending on the order of the harmonic strongly suggests the presence of a hidden Ising-like feature which may correspond to the pseudo-spin variables of the bipolaronic ${ }^{9}$ CDW. Quantitative modeling of these saturations should lead to the spatial extension of the bipolarons. This is under active studies. We are pleased to acknowledge many interesting discussions with J.L. Raimbault, S. Aubry and J.D. Axe.

1 For review see Electronic Properties of Inorganic quasi 1D compounds, Physics and Chemistry of Materials with low dimensional structures, Series B, Edited by P. Monceau, Reidel Publishing Company (1985)

2 A. Fournel et al. Phys. Rev. Lett. $\underline{57}$ 2199, (1986)

3 A.H. Moudden et al Phys. Rev. Lett. $\underline{65}$ 223, (1990)

4 P.A. Lee et al. Phys. Rev. Lett. 31 462, (1973)

5 J.A. Wilson Phys. Rev. B19, 6456 (1979) J.Phys F. 12, 2469, (1982)

6 J.P. Pouget et al J. Physique $\underline{46}, 1731$ (1985) and A.H. Moudden et al. Phys. Rev. B44, 3324 (1991)

7 C. Noguera and J.P. Pouget J. Physique I 11035 (1991)

8 J.L. Raimbault and S. Aubry (1993) (private communication)

9 S. Aubry and P. Quemerais in Low dimensional Electronic Properties of Molybdenum Bronzes Edited by C. Schlenker (Kluwer Academic Publishers) (1989). 